\title{
Interaction of skullcap (Scutellaria baicalensis Georgi) and buckwheat (Fagopyrum esculentum Moench) extracts with lipid bilayers
}

\author{
Hanna Pruchnik ${ }^{1} \cdot$ Aleksandra Włoch $^{1} \cdot$ Romuald Żyłka $^{1} \cdot$ Jan Oszmiański $^{2} \cdot$ \\ Halina Kleszczyńska ${ }^{1}$
}

Received: 20 September 2014/ Accepted: 3 March 2015/Published online: 18 March 2015

(C) The Author(s) 2015. This article is published with open access at Springerlink.com

\begin{abstract}
In this paper, we report the effect of skullcap (Scutellaria baicalensis Georgi) root (SR) and buckwheat (Fagopyrum esculentum Moench) husk and stalk (BH and BS) extracts on lipid organization and fluidity of model membranes, examined by monitoring of the thermotropic phase transition of dimyristoylphosphatidylcholine (DMPC) with differential scanning calorimetry, attenuated total reflection Fourier transform infrared spectroscopy (ATR-FTIR) and fluorescence spectroscopy. Despite the very high biological activity of these extracts, such a study has not been undertaken previously. Calorimetric results on the thermotropic parameters of DMPC membranes suggest that $\mathrm{BH}$ and BS extracts modify them to a greater extent than SR. Fluorescence and ATR-FTIR results confirm that the compounds contained in the SR extract concentrate in the hydrophilic area of the DMPC bilayer, causing an increase in the order of the polar heads of the lipid, and they do not penetrate the hydrophobic area. Compounds contained in the BS and BH extracts also concentrate in the hydrophilic area of the bilayer; however, they partially affect the hydrophobic-hydrophilic interface of the bilayer as well as the upper part of the acyl chain region.
\end{abstract}

Keywords Skullcap - Buckwheat · Phase transition · Lipid bilayers · DSC $\cdot$ ATR-FTIR

Hanna Pruchnik

hanna.pruchnik@up.wroc.pl

1 Department of Physics and Biophysics, Wrocław University of Environmental and Life Sciences, Norwida 25, 50-375 Wrocław, Poland

2 Department of Fruit, Vegetable and Cereal Technology, Wrocław University of Environmental and Life Sciences, ul. Norwida 25, 50-375 Wrocław, Poland

\section{Introduction}

In both the pharmaceutical and food industries, the interest in substances of natural origin is increasing, because such substances are very good antioxidants due to their polyphenolic composition. They reduce or prevent the harmful effects of free radicals in the body and thus inhibit development of certain diseases, such as Alzheimer's disease, Parkinson's disease and cancer [1]. Plants known to have a wide range of health-supporting activity are used to obtain extracts of known polyphenolic composition-those extracts can be used, for example, as nutraceuticals.

Such are the extracts that we have newly obtained from skullcap (S. baicalensis Georgi) root (SR) and buckwheat (Fagopyrum esculentum Moench) husk and stalk (BH and BS). Both plants have been known and used for a very long time, and their composition and therapeutic effects are described in the literature. Thanks to the biological properties of its components [2], the skullcap, in addition to its antioxidant properties [3-5], also has anti-inflammatory $[6,7]$, antimicrobial, antiviral $[8,9]$ and anticancer properties, inhibiting the proliferation of the following tumor cell lines: THP-1 and L1210 murine leukemias, HOS osteosarcoma, SK-MES- 1 of lung cancer and SCC-25 of head and neck cancer [10-13]. Its roots have been used for detoxification of toxins, reducing the total cholesterol level and lowering blood pressure. This herb also possesses cholagogic, diuretic and cathartic actions [6, 14, 15].

Buckwheat also has antioxidant and antibacterial properties [16]. Because buckwheat grains are rich in starch, proteins and fiber, they are added to many foods, such as bread and pasta - in order to increase the healthy properties of the food [17-23]. Buckwheat husk exhibits anticancer properties in relation to different lines of cancer cells [24]. Sprouting seeds can lower blood pressure and protect the 
arterial endothelial cells against oxidative stress [25]. This plant is rich in polyphenols, including the flavonoids rutin, orientin, vitexin, quercetin, isovitexin and isoorientin. In connection with such a wide range of healthy activities, efforts are undertaken to apply the most important ingredients of buckwheat in supportive therapy in many diseases. Among these antioxidant components, rutin was recognized as the most health protective, and it has also been proven to be anti-inflammatory and anticarcinogenic [26, 27].

The surface of the lipid bilayer is the place where processes related to the transfer of information and control of key processes occur, such as cell activation and immunological recognition. Therefore, alteration of lipid bilayer properties may result not only in antioxidant action of flavonoids but also in affecting the activity of integral proteins and hence influence transport and other membrane-related processes [28-41]. The ability of polyphenols to penetrate into lipid bilayers is undoubtedly crucial to the protection against oxidation [42]. It has been suggested [43] that polyphenols that partition in the nonpolar region of the bilayer can inhibit the propagation of lipid oxidation by intercepting intra-membrane radicals and/or by increasing membrane fluidity, which hinders their propagation. Therefore, it is essential to examine the effects of the extracts from the root of skullcap, as well as stalks and husks of buckwheat, on the fluidity of the membrane and to determine the likely location of the polyphenol extract components in the bilayer.

In our earlier study, we demonstrated that these plant extracts are effective in preventing lipid peroxidation in membranes (comparable to or slightly higher than Trolox). Additionally, the tested substances have high anti-inflammatory activity and cause an increase in osmotic resistance of erythrocytes, making them less sensitive to changes in osmotic pressure [44-47].

To complement the above study, the research presented in this paper was conducted, the aim of which was to analyze interactions of extracts of S. baicalensis Georgi (skullcap) and F. esculentum Moench (buckwheat) with the phosphatidylcholine bilayers, and specifically to determine the effect of aforementioned extracts on thermotropic phase behavior of lipids.

The effects of plant extracts on thermotropic phase behavior of lipids were studied using three methods: differential scanning calorimetry (DSC), steady-state fluorimetry and attenuated total reflectance Fourier transform infrared spectroscopy (ATR-FTIR). These methods allowed us to track the changes caused by the action of the polyphenolic extract components in different areas (at different levels) of the bilayer. As a model, we used liposome multi- and unilamellar vesicles formed of dimyristoylphosphatidylcholine (DMPC). This model is very well described in the literature and is known for allowing relatively easy analysis of changes in physical parameters of membrane induced by additional components [28, 29, 34-36].

\section{Experimental}

\section{Materials}

Scutellaria baicalensis Georgi, genus: Scutellaria (skullcap), and F. esculentum Moench, genus: Fagopyrum Mill (buckwheat), were harvested from an experimental field of the Garden of Medicinal Plants Herbarium of the Medical University of Wrocław, Poland. Extracts of skullcap root (SR) and buckwheat husk (BH) and stalk (BS) were obtained from the Department of Fruit, Vegetable and Cereal Technology, Wrocław University of Environmental and Life Sciences. Polyphenols were isolated from skullcap root and buckwheat husk and stalk using the method described by Gąsiorowski et al. [48]. The 1,2-dimyristoyl-snglycero-3-phosphatidylcholine (DMPC) was purchased from Sigma-Aldrich, Steinheim, Germany. The fluorescent probes 6-dodecanoyl-2-dimethylaminonaphthalene (Laurdan), 1,6-diphenyl-1,3,5-hexatriene (DPH) and 1-(4trimethylammoniumphenyl)-6-phenyl-1,3,5-hexatriene $p$ toluenesulfonate (TMA-DPH) were purchased from Molecular Probes, Eugene, Oregon, USA.

\section{Methods}

\section{Preparation of samples}

As the biological membrane model, we used uni- and multilamellar liposomes formed from DMPC in accordance with the following procedure: DMPC was dissolved in chloroform, and then ethanol-dissolved extracts of BS, $\mathrm{SR}$ and $\mathrm{BH}$ were added. Chloroform and ethanol were very carefully evaporated to dryness under nitrogen, and a thin lipid film was formed on the flask wall. Samples were left in a vacuum pump for at least $2 \mathrm{~h}$, after which distilled water was added and the lipid film was washed away from the flask wall using a mechanical shaker. The shaking was conducted at a temperature above the main phase transition of the lipid, until all the lipids created a homogeneous milky suspension of multilamellar vesicles (MLVs) [49].

Small unilamellar liposomes were obtained by sonicating pure DMPC MLVs using a Sonics VCX750 sonicator (Sonics and Materials, Inc.).

\section{Differential scanning calorimetry}

DSC studies were performed as described previously [49]. Samples for DSC were prepared from MLVs of DMPC. 
The final concentration of lipids was $25 \mathrm{mg} \mathrm{cm}^{-3}$. The prepared MLVs of pure lecithin (control sample) and lecithin with extracts were placed in Mettler Toledo standard aluminum crucibles of $40 \mu \mathrm{l}$ capacity. Tightly closed vessels were incubated for 2 days at $4{ }^{\circ} \mathrm{C}$. The measurements were performed with Mettler Toledo Thermal Analysis System D.S.C. $821^{\mathrm{e}}$, operated at a heating rate of $2{ }^{\circ} \mathrm{C} \min ^{-1}$ from 5 to $30{ }^{\circ} \mathrm{C}$. Thermal cycles were repeated three times. The experimental error in temperature and thermal response was $\pm 0.2{ }^{\circ} \mathrm{C}$ and $\pm 5 \%$, respectively. The data were analyzed using original software provided by Mettler Toledo. An empty crucible was used as the reference, and the temperature scale was calibrated with indium.

\section{Fluorescence spectroscopy}

The effect of extracts of SR, BS and BH on phase transition of model membrane was also examined using the fluorimetric method described in our previous work [50, 51], with a few minor modifications. Samples for steady-state fluorimetry consisted of small unilamellar vesicles (SUVs) with DMPC.

Fluorescent probes (Laurdan, DPH and TMA-DPH) were added to SUVs, and after that samples were incubated for $15 \mathrm{~min}$ in darkness at room temperature. Then a solution of extracts was added, and samples were incubated for $30 \mathrm{~min}$

Control samples contained a lipid suspension with a suitable fluorescent probe (concentration of $10 \mu \mathrm{M}$ ), and the appropriate extract (concentration of $0.03 \mathrm{mg} \mathrm{cm}^{-3}$ ) was added to the remaining samples. Measurements were made at different temperatures-above and below the main phase transition of DMPC. Thermal cycles $\left(10-32{ }^{\circ} \mathrm{C}\right)$ were repeated three times.

The measurements were conducted with a CARY Eclipse fluorimeter (VARIAN) equipped with a Peltier temperature controller DBS (temp. accuracy $\pm 0.1{ }^{\circ} \mathrm{C}$ ). The excitation and emission wavelengths for the DPH probe were $\lambda_{\mathrm{ex}}=360 \mathrm{~nm}$ and $\lambda_{\mathrm{em}}=425 \mathrm{~nm}$, whereas for the TMA-DPH probe they were $\lambda_{\mathrm{ex}}=358 \mathrm{~nm}$ and $\lambda_{\mathrm{em}}=428 \mathrm{~nm}$, respectively. The excitation wavelength for Laurdan was $360 \mathrm{~nm}$, and the emitted fluorescence was recorded at two wavelengths: 440 and $490 \mathrm{~nm}$.

Fluorescence anisotropy $(A)$ for the DPH and TMADPH probe was calculated using the formula $A=\left(I_{\mathrm{II}}-\right.$ $\left.G I_{\perp}\right) /\left(I_{\mathrm{II}}+G I_{\perp}\right)$, where $I_{\mathrm{II}}$ and $I_{\perp}$ are fluorescence intensities observed in directions parallel and perpendicular to the polarization direction of the exciting wave, respectively. $G$ is an apparatus constant dependent on emission wavelength. Changes in the polar group packing arrangement of the hydrophilic part of the membrane were investigated using the Laurdan probe, on the basis of generalized polarization (GP), and were calculated with the formula GP $=\left(I_{\mathrm{g}}-I_{1}\right) /\left(I_{\mathrm{g}}+I_{1}\right)$, where $I_{\mathrm{g}}$ and $I_{1}$ are the fluorescence intensities at the gel and fluid phase, respectively $[52,53]$.

\section{Attenuated total reflectance Fourier transform infrared spectroscopy}

The method was applied as described earlier by BonarskaKujawa et al. [38] with a few minor modifications. The experiments were performed with DMPC liposomes (MLVs). IR spectra of a $70 \mathrm{mM}$ lipid suspension were taken. The extract concentration was $8 \mathrm{mg} \mathrm{cm}^{-3}$. Measurements were performed using a Thermo Nicolet $6700 \mathrm{MCT}$ spectrometer (Thermo Fisher Scientific, Waltham, MA) with ZnSe crystal at a heating cycle from 4 to $34{ }^{\circ} \mathrm{C}$. Each single spectrum was obtained from 128 records at $2 \mathrm{~cm}^{-1}$ resolution in the range of $700-4000 \mathrm{~cm}^{-1}$. Preliminary elaboration of a spectrum was done using the EZ OMNIC v 8.0 program, also by Thermo Nicolet. After filtering the noise out of the extract spectrum, the spectrum of the water solution was removed and the baseline was corrected.

In the obtained spectra, we examined four bands:

- 3000-2800 $\mathrm{cm}^{-1}$-vibrations of $\mathrm{CH}_{2}$ and $\mathrm{CH}_{3}$ groups of alkyl chains,

- $1780-1700 \mathrm{~cm}^{-1}$ —corresponding to carbonyl group $(\mathrm{C}=\mathrm{O})$ vibrations,

- $1300-1200 \mathrm{~cm}^{-1}$-vibrations of the phosphate group $\left(\mathrm{PO}_{2}\right)$,

- $1000-940 \mathrm{~cm}^{-1}$ - corresponding to choline group (N$\mathrm{CH}_{3}$ ) vibrations

\section{Results and discussion}

Interaction of skullcap (S. baicalensis Georgi) root (SR) and buckwheat ( $F$. esculentum Moench) husk and stalk (BH and BS) extracts with DMPC membranes was investigated by means of DSC, fluorimetry and infrared spectroscopy.

Using DSC, we examined the heat signal associated with the lamellar gel phase $\left(L_{\beta}\right)$ to ripple phase transition $\left(P_{\beta^{\prime}}\right)-T_{\mathrm{p}}$ (temperature of pretransition) - and with the ripple phase to fluid lamellar phase transition $\left(L_{\alpha}\right)-T_{\mathrm{m}}$ (temperature of main phase transition) - of lipids. In the temperature range $T_{\mathrm{p}}-T_{\mathrm{m}}$, an intermediate phase is observed, where bilayers are modulated by a ripple phase. The effect of SR, BS and BH extracts on the phase transition of DMPC, determined in a DSC experiment, is shown in Fig. 1. The measured transition temperatures and enthalpies for pure DMPC are in good agreement with literature values (Table 1) [54]. 
In the studied range of concentrations (0.01-5 $\mathrm{mg} \mathrm{cm}^{-3}$ ), the investigated extracts practically did not affect the temperature of the main phase transition of MLVs formed from DMPC (Fig. 1; Table 1), but the peak of the main transition of DMPC slightly broadens and becomes asymmetric with increasing concentration of compounds (Fig. 1). Although the differences are not large, it is noticeable that the investigated extracts influence thermotropic parameters of the lipid bilayer in a slightly different way. For the SR extract at higher concentration, the peak of the main transition becomes asymmetric (Fig. 1a)—with a small gibbosity on the right side of the main peak. Such a split of the main peak may suggest that the system is not uniform-there are areas of lower and higher concentration of SR extract components. These results may also indicate the coexistence of various phases with different compositions. Additionally, the enthalpy of the main phase transition marginally decreases, and at the highest concentration of extract, the pretransition is abolished completely. Small shifts of $T_{\mathrm{m}}$ toward higher temperatures with minute changes in $\Delta H$ and increase in $\Delta T_{1 / 2}$ as well as disappearance of the pretransition only at the highest concentration of the extract may suggest that components of SR do not disturb the bilayer and only slightly modify lipid bilayers remaining in the gel phase.

BS and BH extracts, similar to SR extract, do not greatly change the $T_{\mathrm{m}}$, but the enthalpy of the phase transition decreases with their increasing concentration. The main peak broadens and blurs, which is apparent particularly in

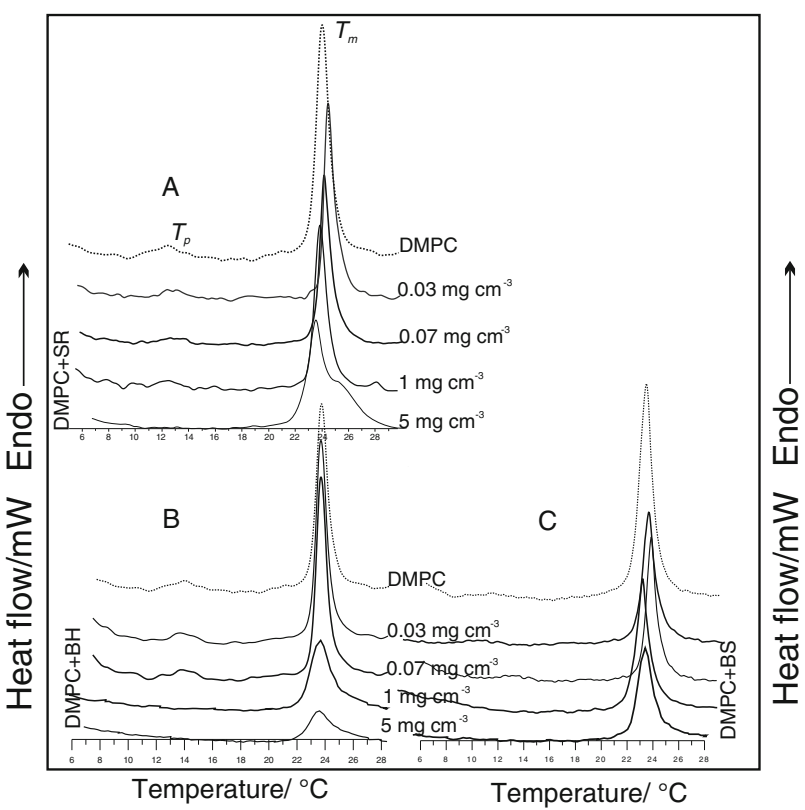

Fig. 1 DSC transition curves of MLVs formed from pure DMPC (dotted line) and DMPC modified with skullcap root extract $(A)$, buckwheat husk $(B)$ and buckwheat stalk $(C)$ extracts. Accuracy of temperature measurement is $\pm 0.2{ }^{\circ} \mathrm{C}$ the case of the $\mathrm{BH}$ extract. At the same time, we observed that this extract abolished the pretransition at a lower concentration than the SR extract (Fig. 1b, c). Since the pretransition is a result of changes in acyl chain packing, its perturbations and/or vanishing indicates that the extracts may influence the structure of the lipid bilayer. On the other hand, compounds that are incorporated into the polar-nonpolar region of the lipid bilayer abolish the DMPC pretransition even at low concentrations [32].

Analyzing the influence of all the extracts on the thermotropic parameters of DMPC membranes, it can be concluded that $\mathrm{BH}$ and $\mathrm{BS}$ extracts modify them to a greater extent than SR, which may suggest that components of SR establish themselves in the vicinity of the polar heads of phospholipids and do not disturb the fluidity of the bilayer.

Similarly small changes in thermotropic parameters were observed for the other extracts investigated by us [38-44].

The effects of SR, BS and BH extracts on phase transition of a model membrane were also analyzed using three probes: Laurdan, TMA-DPH and DPH. These fluorescent probes were used to monitor effects at different bilayer depths and to obtain additional information about the location of the components of SR, BS and BH extracts in DMPC bilayers.

Laurdan becomes located in the hydrophilic-hydrophobic interface of the bilayer. Its fluorophore takes a position near the phospholipid glycerol groups and is sensitive to polarity changes and dynamic properties at the membrane lipid-water interface $[53,55]$. Polarity changes are shown by shifts in the Laurdan emission spectrum, which are quantified by calculating the generalized polarization (GP). When the lipids are in a gel phase, the emission maximum of Laurdan is centered at $440 \mathrm{~nm}$, and when the lipids are in a liquid crystalline phase, the emission maximum is at $490 \mathrm{~nm}$. This spectral shift is the result of a dipolar relaxation of Laurdan to the lipidic environment. The origin of this dipolar relaxation has been attributed to a few water molecules present in the bilayer at the level of the glycerol backbone $[53,55]$. The calculated values of general polarization (GP) for DMPC liposomes as a function of temperature are shown in Fig. 2. Both BS and $\mathrm{BH}$ significantly reduced values of GP. The $\mathrm{BH}$ extract significantly reduced values of GP, BS practically did not change it, whereas SR increased it. The decrease in the GP coefficient indicates that more water molecules are incorporated at the level of the glycerol backbone. This shows that the $\mathrm{BH}$ extract lowers packaging of lipid molecules in the polar range of the bilayer. The effect of the SR extract is opposite: both in the gel phase and in the fluid phase, an increase in the GP coefficient was observed compared to the pure DMPC, which may indicate that components of 
Table 1 Temperature values of the main phase transition $\left(T_{\mathrm{m}}\right)$, peak half-width $\left(\Delta T_{1 / 2}\right)$ and enthalpy change $(\Delta H)$ of the main phase transition for DMPC liposomes, in the absence $\left(0.00 \mathrm{mg} \mathrm{cm}^{-3}\right)$ and in the presence of selected concentrations of SR, BH and BS extracts

\begin{tabular}{|c|c|c|c|c|c|c|c|c|c|}
\hline \multirow[t]{2}{*}{$\mathrm{mg} \mathrm{cm}^{-3}$} & \multicolumn{3}{|l|}{ SR } & \multicolumn{3}{|l|}{$\mathrm{BH}$} & \multicolumn{3}{|l|}{ BS } \\
\hline & $T_{\mathrm{m}} /{ }^{\circ} \mathrm{C}$ & $\Delta T_{1 / 2} /{ }^{\circ} \mathrm{C}$ & $\Delta H / \mathrm{kJ} \mathrm{mol}^{-1}$ & $T_{\mathrm{m}} /{ }^{\circ} \mathrm{C}$ & $\Delta T_{1 / 2} /{ }^{\circ} \mathrm{C}$ & $\Delta H / \mathrm{kJ} \mathrm{mol}^{-1}$ & $T_{\mathrm{m}} /{ }^{\circ} \mathrm{C}$ & $\Delta T_{1 / 2} /{ }^{\circ} \mathrm{C}$ & $\Delta H / \mathrm{kJ} \mathrm{mol}^{-1}$ \\
\hline 0.00 & 24.1 & 0.9 & 26.1 & 24.1 & 0.9 & 26.1 & 24.1 & 0.9 & 26.1 \\
\hline 0.01 & 24.3 & 0.9 & 26.3 & 24.0 & 0.9 & 26 & 24.0 & 0.9 & 20.0 \\
\hline 0.03 & 24.6 & 0.9 & 19.4 & 24.0 & 0.9 & 19.9 & 24.2 & 0.9 & 15.3 \\
\hline 0.05 & 24.4 & 0.9 & 18.2 & 24.1 & 0.9 & 20.5 & 24.2 & 1.0 & 10.9 \\
\hline 1.00 & 23.9 & 0.7 & 18.9 & 23.9 & 1.3 & 14.7 & 24.1 & 1.0 & 12.5 \\
\hline 5.00 & 23.8 & 1.6 & 20.4 & 23.6 & 2.0 & 9.8 & 23.5 & 1.7 & 13.4 \\
\hline
\end{tabular}

Accuracy of temperature measurement is $\pm 0.2{ }^{\circ} \mathrm{C}$

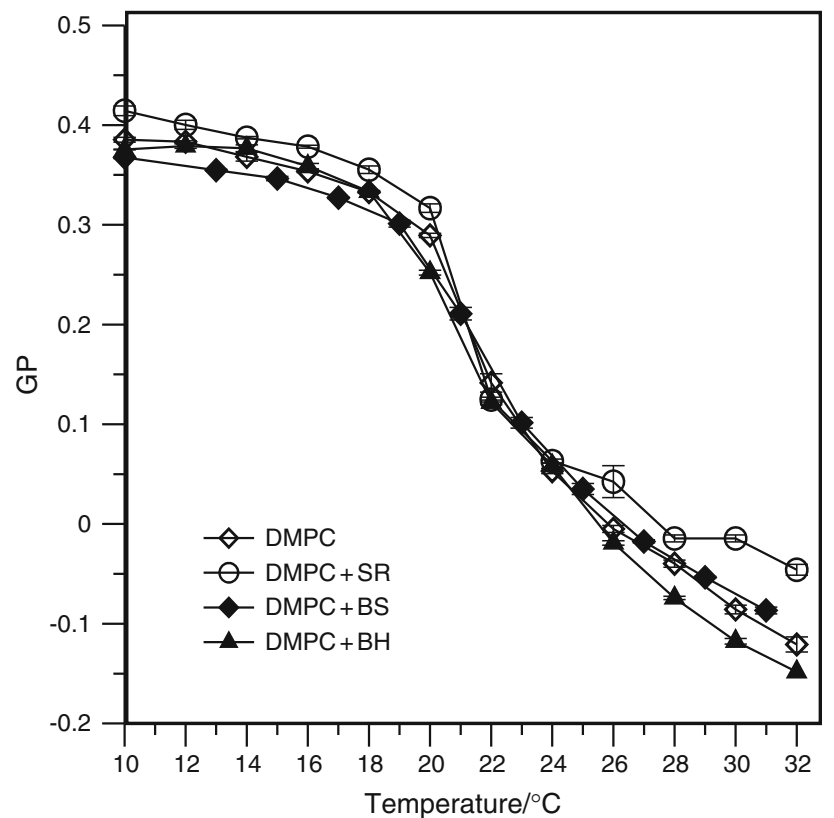

Fig. 2 Values of GP of Laurdan in DMPC SUVs unmodified (control) and modified with SR, BH, BS extracts in concentrations of $0.03 \mathrm{mg} \mathrm{cm}^{-3}$, as a function of temperature

the SR extract locate in the polar range of the bilayer and therefore the bilayer becomes less fluid. These results are in agreement with DSC measurements where a slight increase in $T_{\mathrm{m}}$ was observed at the concentration of $0.03 \mathrm{mg} \mathrm{cm}^{-3}$.

The effect of SR, BS and BH extracts on the fluidity and main phase transition of SUVs formed of DMPC was studied on the basis of anisotropy measured with the DPH and TMA-DPH probes. While DPH is supposed to distribute in the hydrophobic bilayer, TMA-DPH is anchored at the hydrophobic-hydrophilic interface of the bilayer and fixed in the outer region of the acyl chains [56]. Both probes were sensitive to the DMPC phase transition, showing an important decrease in fluorescence anisotropy at temperatures around $24{ }^{\circ} \mathrm{C}$ (Fig. 3). Steady-state fluorescence anisotropy is primarily related to restriction of rotational motion of a dye in the hydrocarbon chain region. Therefore, a decrease in the anisotropy parameter can be explained by a structural perturbation of the bilayer hydrophobic region due to incorporation of a compound $[56,57]$. The dependence of fluorescence anisotropy of the DPH probe on temperature is presented in Fig. 3a. The presence of investigated extracts of $0.03 \mathrm{mg} \mathrm{cm}^{-3}$ concentration practically does not change the temperature of the main phase transition of DMPC. The SR does not change the value of the anisotropy of fluorescence, whereas BH and BS slightly increase this value, but only in the fluid phase. This may indicate a decrease in mobility of the acyl chains. The values of fluorescence anisotropy of TMADPH in SUVs as a function of temperature are presented in Fig. 3b. The SR extract practically does not change $T_{\mathrm{m}}$ of DMPC, but the value of anisotropy slightly increases, both for gel and for fluid phases. This may signify increased ordering of the lipid bilayer. It is interesting that in the presence of BS and BH extracts, the anisotropy of the gel phase diminishes, whereas the anisotropy of the fluid phase fractionally increases, which may suggest that fluidity of the membrane slightly increases at temperatures below $T_{\mathrm{m}}$. These results imply that the investigated compounds, especially BS and BH, significantly influence the fluidity in the hydrophobic region of the lipid bilayer, i.e., in the area of hydrocarbon chains.

The results obtained in studies with fluorescence probes indicate that the compounds contained in the SR extract concentrate in the hydrophilic area of the DMPC bilayer, causing an increase in the order of the polar heads of the lipid, and they do not penetrate the hydrophobic area. Compounds contained in the BS and BH extracts also concentrate in the hydrophilic area of the bilayer, but they partially affect the hydrophobic-hydrophilic interface of the bilayer as well as the upper part of the acyl chain region of the bilayer. 

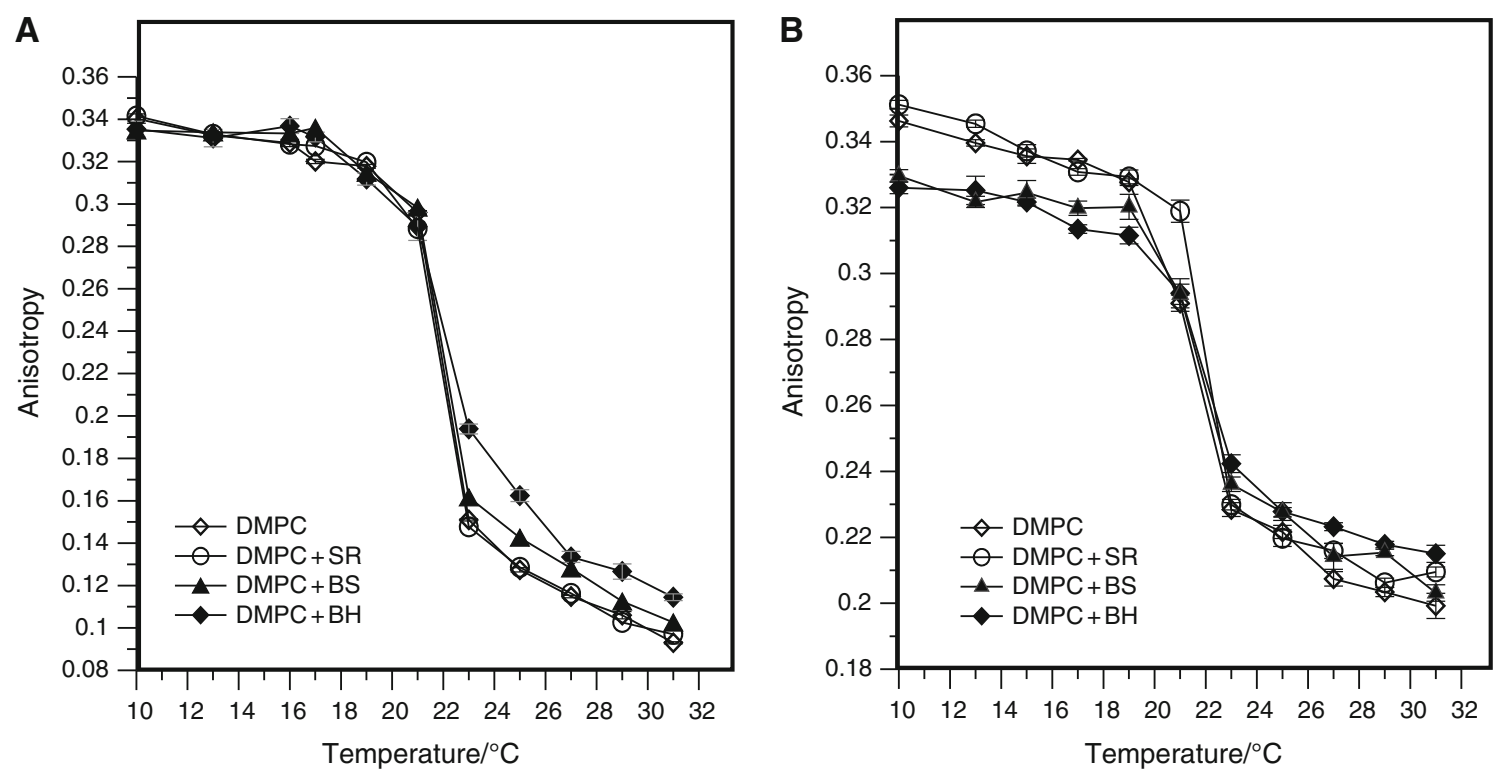

Fig. 3 Fluorescence anisotropy of DPH in SUVs prepared from DMPC (a) and fluorescence anisotropy of TMA-DPH in SUVs prepared from DMPC (b), as a function of temperature

In order to obtain more detailed information about molecular mechanisms of interaction of SR, BS and BH extracts with membranes at higher concentrations of these substances in different phases of DMPC bilayers, the ATRFTIR method was employed.

The infrared spectra of phospholipids can be divided into spectral regions that originate from the molecular vibration of the hydrocarbon tail, the interface region and the head group. The most intense vibrations of lipid systems are the $\mathrm{CH}_{2}$ stretching vibrations, and these give rise to bands in the $3000-2800 \mathrm{~cm}^{-1}$ region. The frequencies of this group of alkyl chains depend on mobility (fluidity) of the chains and increase, e.g., with increasing temperature or during transition from the gel to the liquid crystalline state. The increase in the wavenumber of these bands testifies to an increase in liquidity of the hydrophobic part of the membrane [58-60].

Dependence of the symmetric and asymmetric $\mathrm{CH}_{2}$ stretching vibration in pure DMPC and extract-doped DMPC liposomes as a function of temperature is shown in Fig. $4 a, b$. SR does not change the main phase transition of DMPC; the frequencies of the $\mathrm{CH}_{2}$ group lipid-SR system are practically the same as for the pure lipid for all phases of the bilayer. BS and BH extracts change the frequency of vibrations of methylene groups of acyl chains of DMPC. BS causes an increase in the wavenumber for the ripple $\left(P_{\beta^{\prime}}\right)$ and liquid phases $\left(L_{\alpha}\right)$; thus, the presence of the BS extract slightly lowers $T_{\mathrm{m}}$ and decreases bilayer fluidity, which is in agreement with the DSC results.

The most pronounced changes in frequencies of $\mathrm{CH}_{2}$ of acyl chains of DMPC were observed in the presence of the
BH extract in phases $P_{\beta^{\prime}}$ and $L_{\alpha}$. The value of the wavenumber for $L_{\beta}$ and $P_{\beta^{\prime}}$ is greater than for pure DMPC or DMPC modified with other investigated extracts but lower than for the $L_{\alpha}$ phase. This may indicate that $\mathrm{BH}$ components modify the lipid bilayer in the gel phase by increasing its fluidity and abolishing the sharp phase transition from the gel phase to the liquid crystal phase. This is supported by the broadening (or even blurring) of the main transition peak observed in DSC measurements (Fig. 1b).

Spectral modes arising from the head group and interfacial region can also provide valuable information. The $\mathrm{C}=\mathrm{O}$ band is very useful for probing the interfacial region because in the structure of DMPC, ester groups are located between polar and non-polar interfaces. Any change in the structure of a lipid molecule can be monitored by analyzing this sensitive interfacial region. Examining the $\mathrm{C}=\mathrm{O}$ stretching band provides information about the strength of hydrogen bonding. The changes observed in carbonyl (1760-1700 $\mathrm{cm}^{-1}$ ) bands indicate changes in the degree of hydration of carbonyl groups [58]. In DMPC, there are two bands because of two ester carbonyl groups in the molecule: one around $1742 \mathrm{~cm}^{-1}$ with a trans-conformation in the $\mathrm{C}-\mathrm{C}$ bond and the other one around $1728 \mathrm{~cm}^{-1}$ [58]. However, for hydrated samples, we observed only one broad band contour with a mid-point around $1735 \mathrm{~cm}^{-1}$ and divided into two subcomponents. The low-wavenumber subcomponent reflects the contribution of a subpopulation of hydrogen-bonded carbonyl groups, while the high-wavenumber one arises from free (non-bonded) groups [58]. The relationship of the maximum position of the vibration of the $\mathrm{C}=\mathrm{O}$ band at different temperatures in 

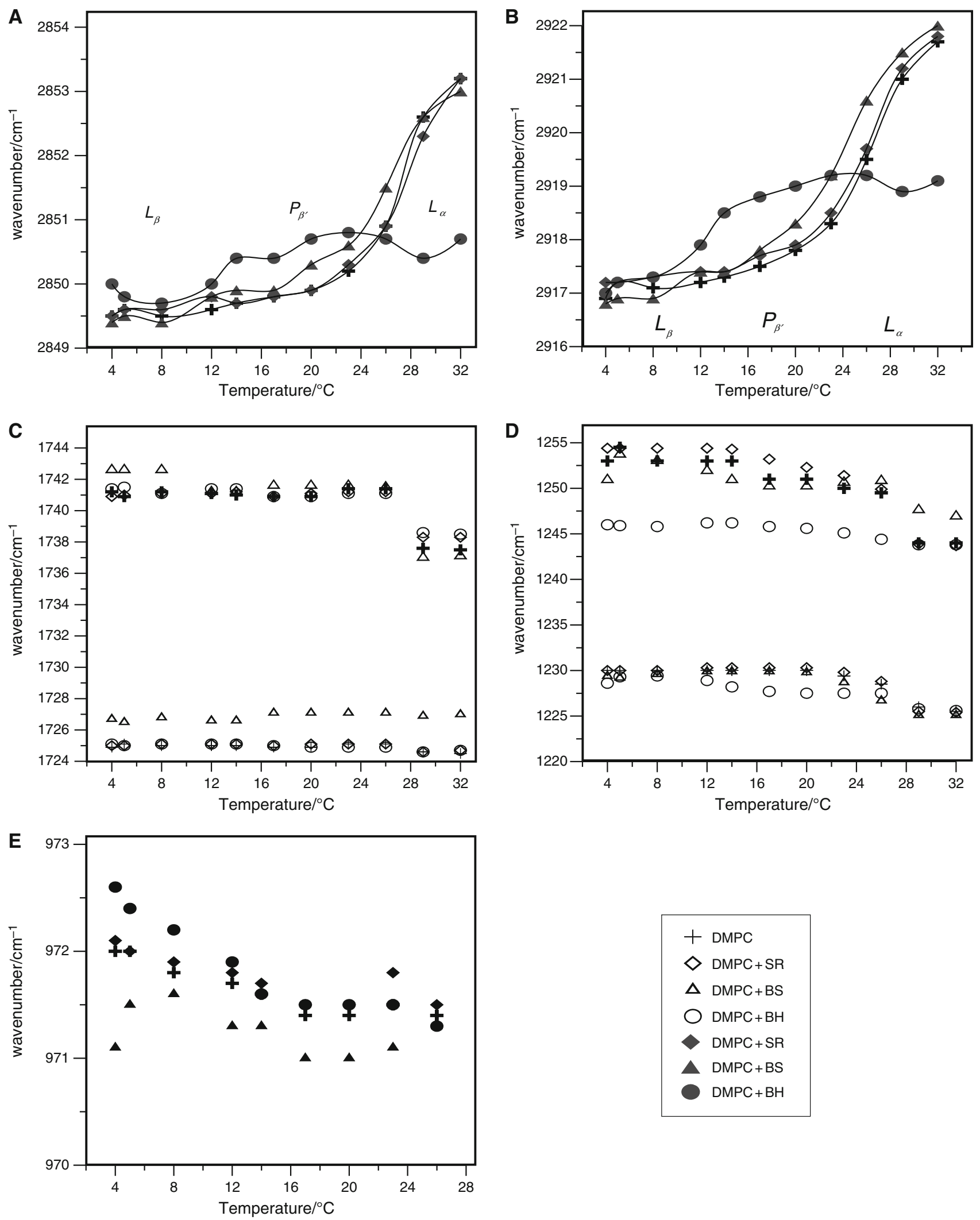

Fig. 4 Position of the symmetric (a) and asymmetric (b) $\mathrm{CH}_{2}$ stretching band as a function of temperature in pure and extract-doped DMPC liposomes, and temperature dependence of the wavenumbers

of the ester $\mathrm{C}=\mathrm{O}$ group $(\mathbf{c})$, the $\mathrm{PO}_{2}$ group $(\mathbf{d})$ and the choline $(\mathrm{N}-$ $\mathrm{CH}_{3}$ ) group (e) stretching in pure and extract-doped DMPC liposomes 
pure and extract-doped DMPC liposomes is shown in Fig. 4c. A slight shift of frequencies of ester groups can be observed for lipids with the BS extract. That change in the spectrum appears to be due to direct interaction between a component in the BS extract and the carbonyl group of lipids and may be the result of hydrogen bond formation between $\mathrm{OH}$ groups of polyphenols and oxygen of the ester bond of lipids [38].

The ester group can bind one molecule of water, while the phosphate group can bind a few. The changes observed in phosphate $\left(1300-1000 \mathrm{~cm}^{-1}\right)$ bands testify, therefore, to changes in the degree of hydration of these groups. The relationship of the maximum position of the vibration of the $\mathrm{PO}_{2}$ band at different temperatures in pure and extractdoped DMPC liposomes is shown in Fig. 4d. Similarly as in the case of the carbonyl group, here we also distinguished two spectrum components attributed to a different degree of hydration of the bilayer. Although the SR extract does not practically change the vibrational frequencies of the $\mathrm{PO}_{2}$ group, it can be observed that the wavenumber is lowered for BH. This shift of phosphate group bands may indicate that water-driven hydrogen bonds are created between those groups and components of the BH extract.

The frequency of vibration of the methyl groups of the choline part (trimethyl ammonium) depends on the medium and grows slightly when its viscosity decreases. In addition, in the band of $\mathrm{N}-\mathrm{C}\left(970 \mathrm{~cm}^{-1}\right)$ vibrations of choline groups, a decrease in the frequencies was observed for BS extract (Fig. 4e), which suggests increased hydration. These results are in agreement with fluorimetric measurements.

Our results obtained with independent methods clearly show that all investigated extracts interact with lipid bilayers. They change the thermotropic properties of liposomes formed from DMPC, but not in the same way. The greatest changes were observed in the case of $\mathrm{BH}$ and $\mathrm{BS}$ extracts. Compounds contained in these extracts not only disturb the polar region of the bilayer but also influence the hydrophobic region, which is particularly apparent for $\mathrm{BH}$. Such effects can be attributed to the fact that the investigated extracts consist of many different compounds, the majority of which are derivatives of quercetin. Our results are in agreement with earlier research on the influence of selected polyphenols, e.g., quercetin, on model membranes [28-30, 36, 41, 61].

In our preliminary examination, it was found that the SR extract is more effective in protecting the erythrocyte membrane against oxidation by UVC than $\mathrm{BH}$ and $\mathrm{BS}$ extracts [44-47]. This may be attributed to the fact that components of the SR extract locate in a different area of the bilayer than components of BS and $\mathrm{BH}$ extracts.

\section{Conclusions}

We have reported the effects of $\mathrm{SR}, \mathrm{BH}$ and $\mathrm{BH}$ extracts on lipid organization and fluidity of model membranes, examined by monitoring the thermotropic phase transition of DMPC with different methods (DSC, ATR-FTIR and fluorescence spectroscopy). To our knowledge, this is the first study to report the interaction of SR, BH and BS with a DMPC model membrane.

All investigated extracts interact with the lipid bilayer. They change the thermotropic properties of liposomes formed from DMPC, although not in the same way: the greatest changes were observed in the case of $\mathrm{BH}$ and $\mathrm{BS}$ extracts. The compounds contained in the SR extract concentrate in the hydrophilic area of the DMPC bilayer, causing an increase in the order of the polar heads of the lipid, and they do not penetrate the hydrophobic area. Compounds contained in the $\mathrm{BS}$ and $\mathrm{BH}$ extracts also concentrate in the hydrophilic area of the bilayer, but they partially affect the hydrophobic-hydrophilic interface of the bilayer as well as the upper part of the acyl chain region of the bilayer.

Acknowledgements The use of a DSC in the Institute of Agricultural Engineering of Wrocław University of Environmental and Life Sciences is gratefully acknowledged. This work was sponsored by the statutory activities of the Department of Physics and Biophysics of Wrocław University of Environmental and Life Sciences.

Open Access This article is distributed under the terms of the Creative Commons Attribution License which permits any use, distribution, and reproduction in any medium, provided the original author(s) and the source are credited.

\section{References}

1. Bullon P, Newman HN, Battino M. Obesity, diabetes mellitus, atherosclerosis and chronic periodontitis: a shared pathology via oxidative stress and mitochondrial dysfunction? Periodontology. 2000;2014(64):139-53.

2. Li HB, Jiang Y, Chen F. Separation methods used for Scutellaria baicalensis active components. J Chromatogr B. 2004;812: 277-90.

3. Dimitrić Marković JM, Marković ZS, Brdarić TP, Pavelkić VM, Jadranin MB. Iron complexes of dietary flavonoids: combined spectroscopic and mechanistic study of their free radical scavenging activity. Food Chem. 2011;129:1567-77.

4. Sawicka E, Średnicka D, Długosz A. Scutellaria baicalensis inhibits lipid peroxidation caused by chromium in human erythrocytes. Adv Clin Exp Med. 2008;17:539-44.

5. Huang WH, Lee AR, Yang ChH. Antioxidative and anti-inflammatory activities of polyhydroxyflavonoids of Scutellaria baicalensis Georgi. Biosci Botechnol Biochem. 2006;70: 2371-80.

6. Li-Weber M. New therapeutic aspects of flavones: the anticancer properties of Scutellaria and its main active constituents Wogonin, Baicalein and Baicalin. Cancer Treat Rev. 2009;35:57-68. 
7. Yoon SB, Lee YJ, Park SK, Kim HC, Bae H, Kim HM, Ko SG, Choi HY, Oh MS, Park W. Anti-inflammatory effects of Scutellaria baicalensis water extract on LPS-activated RAW 264.7 macrophages. J Ethnopharmacol. 2009;125:286-90.

8. Błach-Olszewska Z, Lamer-Zarawska E. Come back to roottherapeutic activities of Scutellaria baicalensis root in aspect of innate immunity regulation-part I. Adv Clin Exp Med. 2008;17:337-45.

9. Hu JZ, Bai L, Chen DG, Xu QT, Southerland WM. Computational investigation of the anti-HIV activity of Chinese medicinal formula Three-Huang Powder. Interdiscip Sci. 2010;2:151-6.

10. Ciesielska E, Gwardys A, Metodiewa D. Anticancer, antiradical and antioxidative actions of novel Antoksyd S and its major components, baicalin and baicalein. Anticancer Res. 2002;22:2885-92.

11. Gao J, Zhao H, Hyland PJ, Corcoran O. Secondary metabolite mapping identifies Scutellaria inhibitors of human lung cancer cells. J Pharm Biomed Anal. 2010;53:723-8.

12. Himeji M, Otsuki T, Fukazawa H, Tanaka M, Yazaki S, Ui S, Nishio K, Yamamoto H, Tasaka K, Mimura A. Difference of growth-inhibitory effect of Scutellaria baicalensis-producing flavonoid wogonin among human cancer cells and normal diploid cell. Cancer Lett. 2007;245:269-74.

13. Zhang DY, Wu J, Ye F, Xue L, Jiang S, Yi J, Yi J, Zhang W, Wei $\mathrm{H}$, Sung M, Wang W, Li X. Inhibition of cancer cell proliferation and prostaglandin E2 synthesis by Scutellaria baicalensis. Cancer Res. 2003;63:4037-43.

14. Tang W, Eisenbrand G. Chinese drugs of plant origin: chemistry, Pharmacology and use in traditional and modern medicine. Berlin: Springer; 1992. p. 919-29.

15. Lu HT, Jiang Y, Chen F. Application of high-speed countercurrent chromatography to the preparative separation and purification of baicalin from the Chinese medicinal plant Scutellaria baicalensis. J Chromatogr A. 2003;1017:117-23.

16. Wang L, Yang X, Qin P, Shan F, Ren G. Flavonoid composition, antibacterial and antioxidant properties of tartary buckwheat bran extract. Ind Crop Prod. 2013;49:312-7.

17. Christa K, Soral-Śmietana M. Buckwheat grains and buckwheat products-nutritional and prophylactic value of their components-a review. Czech J Food Sci. 2008;26(3):153-62.

18. Guo X, Yao H. Fractionation and characterization of tartary buckwheat flour proteins. Food Chem. 2006;98:90-4.

19. Krkošková B, Mrázová Z. Prophylactic components of buckwheat. Food Res Int. 2005;38:561-8.

20. Steadman KJ, Burgoon MS, Lewis BA, Edwardson SE, Obendorf RL. Buckwheat seed milling fractions: description, macronutrient composition and dietary fibre. J Cereal Sci. 2001;33:271-8.

21. Tomotake H, Yamamoto N, Yanaka N, Ohinata H, Yamazaki R, Kayashita J, Kato N. High protein buckwheat flour suppresses hypercholesterolemia in rats and gallstone formation in mice by hypercholesterolemic diet and body fat in rats because of its low protein digestibility. Nutrition. 2006;22(2):166-73.

22. Wronkowska M, Soral-Śmietana M. Buckwheat flour-a valuable component of gluten-free formulations. Pol J Food Nutr Sci. 2008;58:59-63.

23. Zhang ZL, Zhou ML, Tang Y, Li FL, Tang YX, Shao JR, Xue WT, Wu YM. Bioactive compounds in functional buckwheat food. Food Res Int. 2012;49:389-95.

24. Kim SH, Cui CB, Kang IJ, Kim SY, Ham SS. Cytotoxic effect of buckwheat (Fagopyrum esculentum Moench) hull against cancer cells. J Med Food. 2007;10:232-8.

25. Kim DW, Hwang IK, Lim SS, Yoo K-Y, Li H, Kim YS, Kwon DY, Moon WK, Kim D-W, Won M-H. Germinated buckwheat extract decreases blood pressure and nitrotyrosine immunoreactivity in aortic endothelial cells in spontaneously hypertensive rats. Phytother Res. 2009;23:993-8.
26. Liu CL, Chen YS, Yang JH, Chiang BH. Antioxidant activity of tartary (Fagopyrum tataricum (L.) Gaertn.) and common (Fagopyrum esculentum Moench) buckwheat sprouts. J Agr Food Chem. 2008;56:173-8.

27. Liu JL, Tang Y, Xia MZ, Shao JR, Cai GZ, Luo Q, Sun JX. Fagopyrum crispatifolium JL Liu, a new species of Polygonaceae from Sichuan, China. J Syst Evol. 2008;46:929-32.

28. Pawlikowska-Pawlęga B, Misiak LE, Zarzyka B, Paduch R, Gawron A, Gruszecki WI. Localization and interaction of genistein with model membranes formed with dipalmitoylphosphatidylcholine (DPPC). Biochim Biophys Acta. 2012;1818:1785-93.

29. Arora A, Byrem TM, Nair MG, Strasburg GM. Modulation of liposomal membrane fluidity by flavonoids and isoflavonoids. Arch Biochem Biophys. 2000;373:102-9.

30. Sanchez-Galego JI, Lopez-Revuelat A, Sardine JL, HernandezHernandez A, Sanchez-Yague J, Llianillo M. Membrane cholesterol contents modify the protective effects of quercetin and rutin on integrity and cellular viability in oxidized erythrocytes. Free Radic Biol Med. 2010;48:1444-54.

31. Fraga CG, Galleano M, Verstraeten SV, Oteiza PI. Basic biochemical mechanisms behind the health benefits of polyphenols. Mol Aspects Med. 2010;31:435-45.

32. Hendrich $A B$, Michalak K. Lipids as targets for drugs modulating multidrug resistance of cancer cells. Curr Drug Targets. 2003;4:23-30.

33. Pawlikowska-Pawlęga B, Gruszecki WI, Misiak LE, Gawron A. The study of the quercetin action on human erythrocyte membranes. Biochem Pharmacol. 2003;66:605-12.

34. Kużdżał M, Wesołowska O, Štrancar J. Fluorescence and ESR spectroscopy studies on the interaction of isoflavone genistein with biological and model membranes. Chem Phys Lipids. 2011;164:283-91.

35. Wesołowska O, Kużdżał M, Štrancar J, Michalak K. Interaction of the chemopreventive agent resveratrol and its metabolite, piceatannol, with model membranes. Biochim Biophys Acta. 2009;788:1851-60.

36. Hendrich AB, Malon R, Pola A, Shirataki Y, Motohashi N, Michalak K. Differential interaction of Sophora isoflavonoids with lipid bilayers. Eur J Pharm Sci. 2002;16:201-8.

37. Włoch A, Kapusta I, Bielecki K, Oszmiański J, Kleszczyńska H. Activity of hawthorn leaf and bark extracts in relation to biological membrane. J Membr Biol. 2013;246:545-56.

38. Bonarska-Kujawa D, Pruchnik H, Cyboran S, Żyłka R, Oszmiański J, Kleszczyńska H. Biophysical mechanism of the protective effect of blue honeysuckle (Lonicera caerulea L. var. kamtschatica Sevast.) polyphenols extracts against lipid peroxidation of erythrocyte and lipid membranes. J Membr Biol. 2014;247:611-25.

39. Cyboran S, Bonarska-Kujawa D, Pruchnik H, Żyłka R, Oszmiański J, Kleszczyńska H. Phenolic content and biological activity of extracts of blackcurrant fruit. Food Res Int. 2014;65:47-58.

40. Pruchnik H, Bonarska-Kujawa D, Kleszczyńska H. Effect of chlorogenic acid on the phase transition in phospholipid and phospholipid/cholesterol membranes. J Therm Anal Calorim. 2014;118:943-950.

41. Wesołowska O, Gąsiorowska J, Petrus J, Czarnik-Matusiewicz B, Michalak K. Interaction of prenylated chalcones and flavanones from common hop with phosphatidylcholine model membranes. Biochim Biophys Acta. 2014;1838:173-84.

42. Fadel O, El Kirat K, Morandat S. The natural antioxidant rosmarinic acid spontaneously penetrates membranes to inhibit lipid peroxidation in situ. Biochim Biophys Acta. 2011;1808:2973-80.

43. Oteiza PI, Erlejman AG, Verstraeten SV, Keen CL, Fraga CG. Flavonoid-membrane interactions: a protective role of flavonoids at the membrane surface? Clin Dev Immunol. 2005;12:19-25. 
44. Włoch A, Oszmiański J, Kleszczyńska H. Interaction between buckwheat extract and erythrocyte membrane. Eur Biophys J. 2011;40(Suppl 1):199.

45. Włoch A, Kleszczyńska H, Sarapuk J, Bonarska-Kujawa D. Studies on antioxidative efficiency of polyphenols extracted from plants. Eur Biophys J. 2009;38(Suppl 1):196.

46. Kleszczyńska H, Bonarska-Kujawa D, Włoch A, Tomicki B, Oszmiański J. Polyphenol compounds as effective antioxidants. Eur Biophys J. 2007;36(Supl 1):75.

47. Włoch A, Bonarska-Kujawa D, Jessa K, Oszmiański J, Kleszczyńska H. Antioxidative activity of polyphenols and their influence on properties of erythrocyte membranes. Acta Biochim Pol. 2008;55(Suppl 3):270.

48. Gąsiorowski K, Szyba K, Brokos B, Kołaczyńska B, JankowiakWłodarczyk M, Oszmiański J. Antimutagenic activity of anthocyanins isolated from Aronia melanocarpa fruits. Cancer Lett. 1997;119:37-46.

49. Dua JS, Rana AC, Bhandari AK. Liposome: methods of preparation and applications. IJPSR. 2012;III(Issue II):14-20.

50. Pruchnik H, Kral T, Hof M. Interaction of new butyltin citrate complex with lipid model membrane and DNA. J Therm Anal Calorim. 2014;118:967-975.

51. Bonarska-Kujawa D, Pruchnik H, Kleszczyńska H. Interaction of selected anthocyanins with erythrocytes and liposome membranes. Cel Mol Biol Lett. 2012;17:289-308.

52. Lakowicz JR (2006) Fluorescence polarization. In: Principles of fluorescence spectroscopy. Plenum Press, New York, pp 353-382.

53. Parasassi T, Krasnowska EK, Bagatolli L, Gratton E. Laurdan and Prodan as polarity-sensitive fluorescent membrane probes. J Fluoresc. 1998;8:365-73.
54. Blume A. Biological calorimetry: membranes. Therm Acta. 1991;193:299-347.

55. Parasassi T, De Stasio G, Ravagnan G, Rusch RM, Gratton E. Quantitation of lipid phases in phospholipid vesicles by the generalized polarization of Laurdan fluorescence. Biophys J. 1991;60:179-89.

56. Engelke M, Bojarski P, Blo $\beta$ R, H Diehl. Tamoxifen perturbs lipid bilayer order and permeability: comparison of DSC, fluorescence anisotropy, Laurdan generalized polarization carboxyfluorescein leakage studies. Biophys Chem. 2001;90:157-73.

57. Dumas D, Muller S, Gouin F, Baros F, Viriot M-L, Stoltz JF. Membrane fluidity and oxygen diffusion in cholesterol-enriched erythrocyte membrane. Arch Biochem Biophys. 1997;341:34-9.

58. Lewis RNAH, McElhaney RN, Pohle W, Mantsch HH. Components of the carbonyl stretching band in the infrared spectra of hydrated 1,2-diacylglycerolipid bilayers: a reevaluation. Biophys J. 1994;64:2367-75.

59. Attar M, Kates M, Khalil MB, Carrier D, Wong PTT, Tanphaichitr N. A Fourier-transform infrared study of the interaction between germ-cell specific sulfogalactosylglycerolipid and dimyristoylglycerophosphocholine. Chem Phys Lipids. 2000;106:101-14.

60. Goormaghtigh E, Raussens V, Ruysschaert JM. Attenuated total reflection infrared spectroscopy of proteins and lipids in biological membranes. Biochim Biophys Acta. 1999;1422:105-85.

61. Cieślik-Boczula K, Maniewska J, Grynkiewicz G, Szeja W, Koll $\mathrm{K}$, Hendrich $\mathrm{AB}$. Interaction of quercetin, genistein and its derivatives with lipid bilayers - an ATR IR-spectroscopic study. Vib Spectrosc. 2012;62:64-9. 